\title{
Méthodes d'interaction fluide-structure pour la simulation multi-échelles des procédés de mélange
}

\author{
Rudy Valettea ${ }^{a}$, Julien Bruchon, Hugues Digonnet, Patrice Laure, \\ Mathilde Lebceuf, Luisa Silva, Bruno Vergnes et Thierry Coupez \\ Centre de Mise Forme des Matériaux, École Nationale Supérieure des Mines de Paris, UMR CNRS 7635, BP 207, \\ 06904 Sophia Antipolis Cedex, France
}

\begin{abstract}
Reçu le 15 mars 2007, accepté le 7 juin 2007
Résumé - Nous présentons une méthode d'interaction fluide-structure basée sur une approche monolithique eulérienne permettant d'étudier à différentes échelles les procédés de mélange sous leur aspect dispersif et distributif. Une première approche macroscopique traitant de la résolution mécanique dans le procédé, où interviennent des outils tournants, est présentée, puis une approche microscopique modélisant la dispersion d'un agglomérat, et enfin une approche couplée via une théorie cinétique.
\end{abstract}

Mots clés : Calcul multiéchelle / mélange distributif et dispersif / méthode éléments-finis / interaction fluide-structure

\begin{abstract}
Fluid-structure interaction methods for multiscale simulation of mixing processes. A fluid-structure interaction method, based on a eulerian monolithic approach is introduced in order to study distributive and dispersive aspects of mixing within a multiscale approach of the processes. A first example investigates macroscopic flow resolution for the whole process including moving tools, then the dispersion of a single agglomerate is studied within a microscopic approach, and finally one shows a full macroscopic simulation coupled through a kinetic theory for dispersive mixing.
\end{abstract}

Key words: Multiscale computation / distributive and dispersive mixing / finite-elements method / fluid-structure interaction

\section{Introduction}

On présente un ensemble de méthodes de calcul numérique d'interactions fluide-structure que l'on applique à l'étude multi-échelles des procédés de mélange dans les polymères fondus. Ces méthodes numériques sont développées dans la bibliothèque d'objets $\mathrm{C}++$ pour les éléments-finis CIMlib du Centre de Mise en Forme des Matériaux. L'approche proposée repose sur une méthode commune au calcul des champs de vitesse et pression macroscopiques du fluide induits par le mouvement d'outils mélangeurs, (rotors de mélangeur interne, éléments d'extrudeuse bi-vis, pales de malaxeurs, Fig. 1) et aux phénomènes microscopiques d'érosion et rupture d'agglomérats sous l'effet d'un taux de déformation de leur matrice fluide (Fig. 2). Cette méthode, de type monolithique, utilise les éléments-finis associés à une formulation multi-domaines pour le fluide visqueux et les particules ou mélangeurs solides.

\footnotetext{
a Auteur correspondant : rudy.valette@ensmp.fr
}

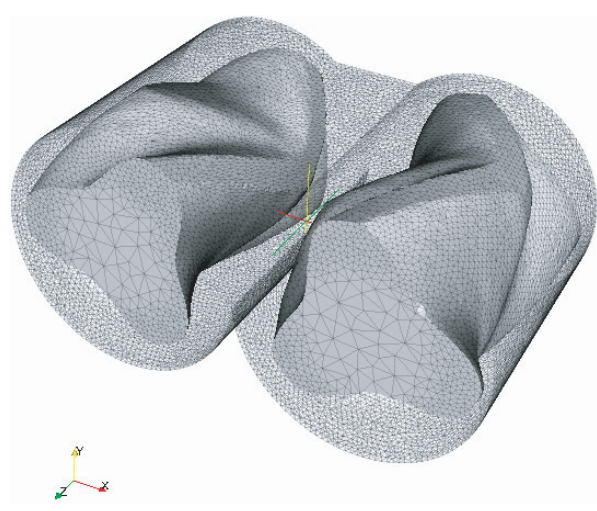

Fig. 1. Représentation, dans le maillage de calcul, de la surface des rotors d'un mélangeur interne par isovaleur à zéro de la fonction level set de chaque domaine solide représentant un rotor.

Dans la suite, on présente l'approche développée pour décrire ces domaines, basée sur l'approximation level set. Le déplacement des particules/objets est effectué 


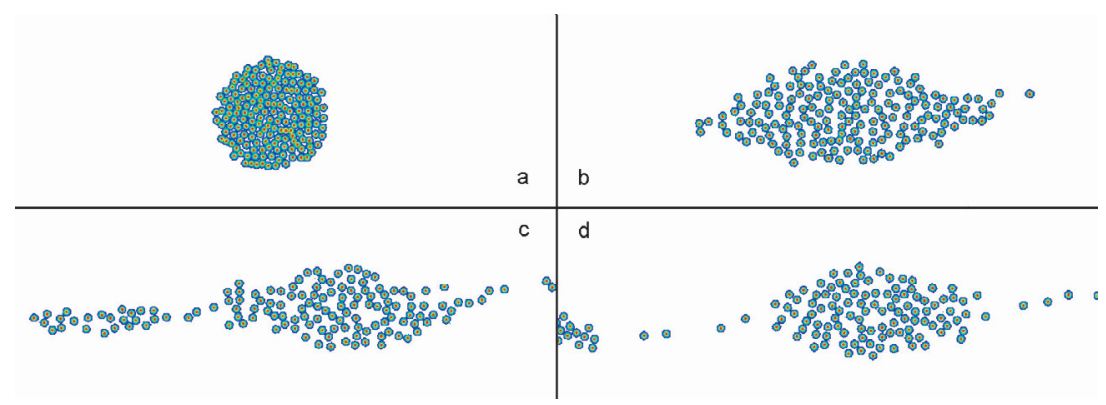

Fig. 2. Représentation (partie intérieure de la fonction distance) de 178 sous-domaines solides sphériques constituant un agglomérat de charges, soumis à un taux de cisaillement de $5 \mathrm{~s}^{-1}$ dans un entrefer de $5 \mathrm{~mm}$.

en utilisant une méthode particulaire qui assure un déplacement de corps rigide et une conservation du domaine solide. Cette formulation s'inspire de la méthode des domaines fictifs utilisée dans $[1,2]$ pour des sphères.

\section{Une classification des approches multi-domaines}

Il existe deux grandes approches différentes pour résoudre les problèmes de type fluide-solide. Dans le problème à traiter existent a priori deux sous-problèmes couplés : la résolution de l'écoulement sur le domaine fluide variable (donc un calcul de champ de vitesse eulérien), et la dynamique d'objets solides plongés dans le fluide (donc un calcul de vitesses lagrangiennes). Les deux manières distinctes d'aborder ces problèmes sont les suivantes.

\subsection{Méthode de maillage du domaine fluide}

Cette méthode repose sur une description « exacte $»$ du domaine fluide, proposée par $\mathrm{Hu}$ [3]. Après résolution du champ de vitesse fluide, les efforts sur les particules sont calculés pour obtenir leur vitesse, puis leur déplacement. Le domaine fluide est alors remaillé en utilisant par exemple des formulations de type ALE (arbitrairement eulérienne lagrangienne), avant d'effectuer le calcul au pas de temps suivant. Ce type de méthode présente deux inconvénients, qui se traduisent par un alourdissement considérable du temps de calcul. D'une part, le maillage est déplacé à chaque pas de temps et, d'autre part, le domaine à mailler est de géométrie complexe et empêche donc l'utilisation de maillages réguliers.

\subsection{Méthode de maillage du domaine total}

Dans ce type de méthode, le problème faible à résoudre est étendu du domaine fluide au domaine total, en prolongeant le champ de vitesse au domaine solide. Un problème fluide est ainsi résolu sur tout le domaine « fictif » fluidesolide. Glowinski et al. [4] furent les premiers à utiliser cette technique. De cette manière, la condition de rigidité des particules n'est plus explicite et il convient de la rajouter dans la formulation faible. L'intérêt des méthodes par domaine fictif est que le calcul du champ de vitesse eulérien se fait sur le domaine total, qui a une géométrie a priori simple et qui est fixe. Le maillage est donc fixe et ne doit être calculé qu'une seule fois. Dans cette approche, la stratégie numérique va être induite par la manière de résoudre les problèmes suivants :

- la représentation du domaine solide,

- le calcul du champ de vitesse,

- le déplacement du domaine solide.

Ces trois méthodes sont présentées dans leur généralité dans la suite, puis les applications spécifiques au mélange sont détaillées : résolution mécanique macroscopique dans un mélangeur interne, étude de la dispersion d'un agglomérat de particules et, enfin, couplage entre théorie cinétique et résolution macroscopique dans le mélangeur interne.

\section{Caractérisation des domaines fluide et solide}

Dans la suite, les champs de vitesse seront définis dans l'espace de Sobolev $H^{1}(\Omega)$, la pression dans $L^{2}(\Omega)$, l'espace de Lebesgue des fonctions de carrées sommable sur $\Omega$. On notera le produit scalaire sur $L^{2}(\Omega)$ par :

$$
\left(f_{1}, f_{2}\right)=\int_{\Omega} f_{1} f_{2} \mathrm{~d} \Omega
$$

Finalement, on utilisera le symbole h pour se référer à l'espace de discrétisation $\Omega_{\mathrm{h}}$ qui est formé par l'union de tous les simplexes (ou éléments) K qui constituent le maillage du domaine $\Omega$. Le domaine de calcul $\Omega$ est décomposé en deux sous domaines, $\Omega_{\mathrm{f}}$ et $\Omega_{\mathrm{s}}$, associés respectivement à la partie fluide et solide. Notons que, lorsque le domaine solide $\Omega_{\mathrm{s}}$ est constitué de plusieurs particules, il vient :

$$
\Omega_{\mathrm{s}}=\sum_{i=1}^{n} \Omega_{\mathrm{S}_{\mathrm{i}}}
$$

pour $n$ particules. La formulation variationnelle faisant intervenir des intégrales sur tout le domaine de calcul, et 


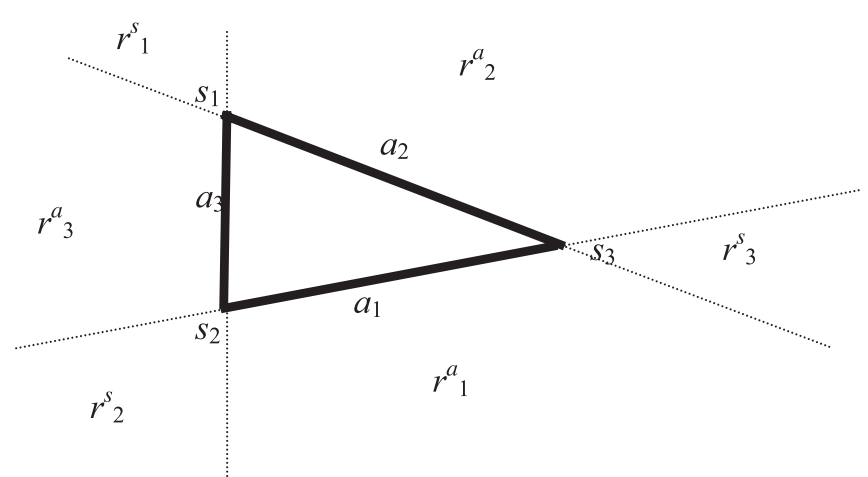

Fig. 3. Notation des différents sommets, arêtes et zones de projection d'un point de l'espace sur son plan pour un triangle.

pour différencier les deux domaines dans le calcul de ces intégrales, on introduit des fonctions caractéristiques $I_{\mathrm{j}}$, $j \in\{f, s\}$ associées à chaque domaine, $\Omega_{\mathrm{j}}, j \in\{f, s\}$, avec :

$$
I_{j}(x, t)=1, \text { si } x \in \Omega_{j} \text { et } I_{j}(x, t)=0 \text { si } x \notin \Omega_{j}
$$

On considérera dans la suite la fonction caractéristique $I_{\mathrm{S}}$ associée au seul domaine solide pour simplifier les notations. L'approximation de la fonction caractéristique adoptée dans ce travail utilise la méthode level set.

\subsection{La fonction distance ou level set}

La fonction caractéristique définie par (2) est approximée par l'intermédiaire d'une fonction régulière d'approximation d'ordre $P_{1}$. Le principe est de définir une fonction distance signée dans le domaine de calcul dont l'isovaleur zéro est l'interface que l'on cherche à déterminer. Cette fonction est choisie positive à l'intérieur d'un sous-domaine et négative partout ailleurs.

Si l'interface du domaine solide est donnée par une courbe $\Gamma_{\mathrm{s}}$, on peut définir la fonction level set $\alpha$ par :

$$
\left\{\begin{array}{l}
\alpha(x)=\left\|x-\Gamma_{\mathrm{s}}\right\| \text { dans } \Omega_{\mathrm{s}} \\
\alpha(x)=-\left\|x-\Gamma_{\mathrm{s}}\right\| \text { dans } \Omega_{\mathrm{f}}
\end{array}\right.
$$

Pour donner une approximation $P_{1}$ de cette fonction, il suffit de l'évaluer aux nœuds du maillage. Une approximation $P_{1}$ de la fonction caractéristique s'obtient alors simplement en évaluant le signe de $\alpha_{\mathrm{h}}\left(x_{\mathrm{i}}\right)$ aux nœuds $x_{\mathrm{i}}$ du maillage :

$$
I_{\mathrm{s}}^{\mathrm{h}}\left(x_{\mathrm{i}}\right)=\left\{\begin{array}{l}
1 \text { si } \alpha_{\mathrm{h}}\left(x_{\mathrm{i}}\right)>0 \\
0 \text { si } \alpha_{\mathrm{h}}\left(x_{\mathrm{i}}\right)<0
\end{array}\right.
$$

Une transition plus régulière peut être obtenue en utilisant par exemple :

$$
I_{\mathrm{S}}=\frac{1}{1+\mathrm{e}^{-\beta \alpha}}, \beta>0
$$

où la zone de transition dépendra de la valeur de $\beta$. Finalement, une approximation $P_{0}$ de la fonction caractéristique à partir de la fonction distance $\alpha$ peut être approximée par :

$$
\left.I_{\mathrm{s}}^{\mathrm{h}}\right|_{\mathrm{K}}=\frac{\alpha_{\mathrm{K}}^{+}}{|\alpha|_{\mathrm{K}}}
$$

où $\alpha_{\mathrm{K}}^{+}$est la somme des $\alpha$ positifs aux nœuds de l'élément $\mathrm{K}$ et $|\alpha|_{\mathrm{K}}$ est la somme des valeurs absolues de $\alpha$. Cette fonction caractéristique intervient dans les relations de mélange qui donnent les valeurs de la viscosité et de la densité sur tout le maillage, comme décrit en section 4 .

\subsection{Calcul de distance à des objets complexes}

Le calcul de la fonction distance $\alpha$ à des objets de formes complexes tels que des éléments mélangeurs présentés à la figure 1 nécessite un traitement particulier. En effet, dans la phase de pré-calcul, la surface $\Gamma_{\mathrm{s}}$ de ces objets complexes n'est pas définie, dans le cas général, par une formule analytique mais par une représentation approchée, telle qu'une triangulation STL (stéréolithographique) générée par des logiciels de conception assistée par ordinateur.

Le calcul de la fonction distance d'un point $x$ du maillage à la surface de l'objet revient alors à un calcul du minimum des distances de ce point à chaque triangle de la surface.

Cette distance est définie par la distance à la région intérieure au triangle, la distance à l'arête $a_{\mathrm{i}}$ du triangle ou la distance au sommet $s_{\mathrm{j}}$ du triangle, selon que le projeté du point $x$ sur le plan défini par le triangle est, respectivement, dans la région intérieure au triangle, la région $r_{\mathrm{i}}^{\mathrm{a}}$, ou la région $r_{\mathrm{j}}^{\mathrm{s}}$ comme défini à la figure 3 .

La recherche de la distance minimale est accélérée par un pré-partitionnement hiérarchique, sous forme d'octree, de l'espace occupé par le maillage triangulé dans le maillage de calcul. Les détails du calcul et de l'algorithme sont donnés dans [5]. Dans la suite, on utilisera le terme d' «immersion de maillage » pour se référer à cette méthode.

\section{Résolution du problème en vitesse-pression}

Dans la formulation multi-domaine, les équations décrivant le comportement du fluide s'écrivent, pour un fluide qu'on choisit ici newtonien pour simplifier les notations :

$$
\begin{cases}\rho_{\mathrm{f}}\left(\frac{\partial \vec{u}}{\partial t}+\vec{u} \cdot \nabla \vec{u}\right) & =\rho_{\mathrm{f}} \vec{g}+\nabla \cdot \underline{\underline{\sigma}} \\ \nabla \cdot \vec{u} & =0 \\ \underline{\underline{\sigma}} & =-p \underline{\underline{I}}+2 \eta_{\mathrm{f}} \underline{\underline{\dot{\varepsilon}}}(\vec{u}) \\ {[[\vec{u}]]_{\Gamma \mathrm{s}}} & =0 \\ [\underline{\underline{\sigma}} \cdot \vec{n}]]_{\Gamma \mathrm{s}} & =0 \\ \vec{u} & =\vec{u}_{\Gamma} \text { sur la frontière extérieure } \Gamma\end{cases}
$$


où $\vec{u}$ est la vitesse, $\mathrm{p}$ la pression, $\eta_{\mathrm{f}}$ la viscosité du fluide, $\rho_{\mathrm{f}}$ sa densité et $[[.]]_{\Gamma s}$ le saut de « . » à travers l'interface fluide-solide $\Gamma_{\mathrm{s}}$. Patankar et al. [6] ont proposé d'étendre les équations de Navier-Stokes au domaine solide à l'aide de multiplicateurs de Lagrange, en utilisant la condition de rigidité $\underline{\underline{\varepsilon}}(\vec{u})=0$ sur $\Omega_{\mathrm{s}}$. On peut ainsi exprimer que, dans le domaine solide $\Omega_{\mathrm{s}}$, le mouvement est celui d'un fluide soumis à une contrainte additionnelle de rigidité :

$$
\begin{cases}\rho_{\mathrm{s}}\left(\frac{\partial \vec{u}}{\partial t}+\vec{u} \cdot \nabla \vec{u}\right) & =\rho_{\mathrm{s}} \vec{g}+\nabla \cdot \underline{=} \\ \nabla \cdot \vec{u} & =0 \\ \underline{\dot{\varepsilon}}(\vec{u}) & =0 \\ \overline{[}[\vec{u}]]_{\Gamma \mathrm{s}} & =0 \\ {[[\underline{\underline{\sigma}} \cdot \vec{n}]]_{\Gamma \mathrm{s}}} & =0\end{cases}
$$

$\rho_{\mathrm{s}}$ étant la masse volumique du corps solide. Cela revient à chercher un tenseur des contraintes $\underline{\underline{\sigma}}$ dans le solide sous la forme :

$$
\underline{\underline{\sigma}}=-p \underline{\underline{I}}+2 \eta_{\mathrm{s}} \underline{\underline{\dot{\varepsilon}}}(\vec{u})+\underline{\underline{\tau}}
$$

sous la contrainte de mouvement de corps rigide $\underline{\underline{\varepsilon}}(\vec{u})=0$ dont $\eta_{\mathrm{s}}$ est un facteur de pénalisation et à laquelle le tenseur symétrique $\underline{\underline{\tau}}$ est le multiplicateur de Lagrange associé. Il a cependānt été montré par Laure et al. [7] que le calcul du multiplicateur de Lagrange n'améliore la précision du calcul que dans le cas de maillages très fins, en pratique peu utilisés en $3 \mathrm{D}$, dès qu'un facteur de pénalisation $\eta_{\mathrm{s}}$ de l'ordre de $10^{3} \eta_{\mathrm{f}}$ est utilisé. Par conséquent, la méthode proposée par Patankar et al. [6] est ici ramenée à une méthode par pénalisation, que l'on décrit dans la suite.

\subsection{Formulation variationnelle du système fluide solide}

Sous l'hypothèse de conditions aux limites de Dirichlet sur les parois extérieures, le problème variationnel sur tout le domaine de calcul $\Omega$ s'écrit alors :

Trouver $(\vec{u}, p)$ tel que $\forall(\vec{v}, q) \in H_{0}^{1}(\Omega) \times L^{2}(\Omega)$ :

$$
\left\{\begin{array}{l}
\int_{\Omega} 2 \eta\left(I_{\mathrm{s}}\right) \underline{\underline{\dot{\varepsilon}}}(\vec{u}): \underline{\underline{\dot{\varepsilon}}}(\vec{v}) \mathrm{d} \Omega-\int_{\Omega} p \nabla \cdot \vec{v} \mathrm{~d} \Omega \\
+\int_{\Omega} \rho\left(I_{\mathrm{S}}\right)\left(\frac{\mathrm{d} \vec{u}}{\mathrm{~d} t}-\vec{g}\right) \cdot \vec{v} \mathrm{~d} \Omega=0 \\
\int_{\Omega} q \nabla \cdot \vec{u} \mathrm{~d} \Omega=0
\end{array}\right.
$$

où $\rho$ et $\eta$ sont définis sur tout le domaine par une loi de mélange :

$$
\rho\left(I_{\mathrm{s}}\right)=I_{\mathrm{s}} \rho_{\mathrm{s}}+\left(1-I_{\mathrm{s}}\right) \rho_{\mathrm{f}} ; \eta\left(I_{\mathrm{s}}\right)=I_{\mathrm{s}} \eta_{\mathrm{s}}+\left(1-I_{\mathrm{s}}\right) \eta_{\mathrm{f}}
$$

\subsection{Formulation discrète et algorithme utilisé}

Pour résoudre le problème non-linéaire et l'intégration en temps, on a recours à un schéma en temps implicite et à une méthode de Newton dont l'algorithme est décrit par :

À chaque pas de temps $t_{n}$, opérer :

1. Initialisation par les valeurs obtenues au pas de temps précédent :

$$
\vec{u}_{\mathrm{h}}^{0}=\vec{u}_{\mathrm{h}}\left(t_{n-1}\right) ; p_{\mathrm{h}}^{0}=p_{\mathrm{h}}\left(t_{n-1}\right) ; \vec{u}_{\mathrm{h}}^{*}=\vec{u}_{\mathrm{h}}\left(t_{n-1}\right)
$$

2. À l'étape $k$, calcul de $\vec{u}_{\mathrm{h}}^{\mathrm{k}}$ et $p_{\mathrm{h}}^{\mathrm{k}}$ en résolvant le système :

$\left\{\begin{array}{l}\left(\rho \frac{\vec{u}_{\mathrm{h}}^{k}}{\Delta t}, \vec{v}_{\mathrm{h}}\right)+\left(\rho \vec{u}_{\mathrm{h}}^{k} \cdot \vec{u}_{\mathrm{h}}^{k-1}, \vec{v}_{\mathrm{h}}\right)+2\left(\eta \underline{\underline{\varepsilon}}\left(\vec{u}_{\mathrm{h}}^{k}\right): \underline{\underline{\varepsilon}}\left(\vec{v}_{\mathrm{h}}\right)\right) \\ -\left(p_{\mathrm{h}}^{k}, \nabla \cdot \vec{v}_{\mathrm{h}}\right)=\left(\rho \vec{g}, \vec{v}_{\mathrm{h}}\right)+\left(\rho \frac{\vec{u}_{\mathrm{h}}^{*}}{\Delta t}, \vec{v}_{\mathrm{h}}\right)+\left(\rho \vec{u}_{\mathrm{h}}^{k-1} \cdot \vec{u}_{\mathrm{h}}^{k-1}, \vec{v}_{\mathrm{h}}\right) \\ \left(\nabla \cdot \vec{u}_{\mathrm{h}}^{k}, q_{\mathrm{h}}\right)=0\end{array}\right.$

3. Test d'arrêt $\left\|\vec{u}_{\mathrm{h}}^{k-1}-\vec{u}_{\mathrm{h}}^{k}\right\|<\varepsilon_{1}$ de la boucle sur $k$.

La résolution du problème utilise ici l'élément mixte $P_{1}+/ P_{1}$, dans lequel la vitesse et la pression sont interpolées linéairement, et une fonction bulle est ajoutée pour enrichir l'espace fonctionnel en vitesse et ainsi vérifier le condition inf-sup [8]. Chaque problème linéaire de l'étape $k$ est résolu par une méthode de résidu conjugué associée à un pré-conditionneur ILU de la bibliothèque parallèle PETSC (Portable, Extensible Toolkit for Scientific Computation). La stratégie parallèle employée, détaillée dans [9], est de type maître-esclave par modules $S P M D$ (single program, multiple data) et utilise le standard de la bibliothèque MPI (Message Passing Interface).

\section{Transport des domaines solides}

À l'issue de la résolution du problème discret, le calcul des nouvelles positions des domaines solides est requis avant l'itération suivante du schéma temporel. Il est donc nécessaire de résoudre l'équation de transport :

$$
\frac{\partial I_{\mathrm{s}}}{\partial t}+(\vec{u} . \nabla) I_{\mathrm{s}}=0
$$

qui traduit la conservation de la masse totale de solide contenue dans le domaine $\Omega_{\mathrm{s}}$. Dans le cadre de ce travail, on a privilégié l'utilisation d'une méthode de déplacement lagrangien à une résolution de l'équation de transport du champ $I_{\mathrm{s}}$ sur tout le maillage.

En effet, dans le cas de la résolution macroscopique d'un procédé de mélange, la position des outils mobiles peut être déterminée explicitement, connaissant leur vitesse de rotation. La procédure décrite à la section 3.2. est alors effectuée systématiquement à chaque pas de temps.

Dans le cas de particules sphériques, dont le champ de vitesse n'est pas connu a priori, il faut calculer le déplacement lagrangien de leur centre à partir du champ de vitesse calculé, puis d'effectuer une mise à jour du champ level set $\alpha$.

Dans cette méthode, le déplacement d'un point s'effectue ainsi à l'aide de la relation suivante :

$$
\vec{X}(t+\Delta t)=\vec{X}(t)+\Delta t \cdot \vec{u}(\vec{X})
$$

où $\vec{u}(\vec{X})$ est la vitesse du centre de la particule. Une méthode hiérarchique du même type que présentée à la section 3.2. est alors utilisée pour interpoler le vecteur $\vec{u}(\vec{X})$ à partir du champ de vitesse solution du problème mécanique. 


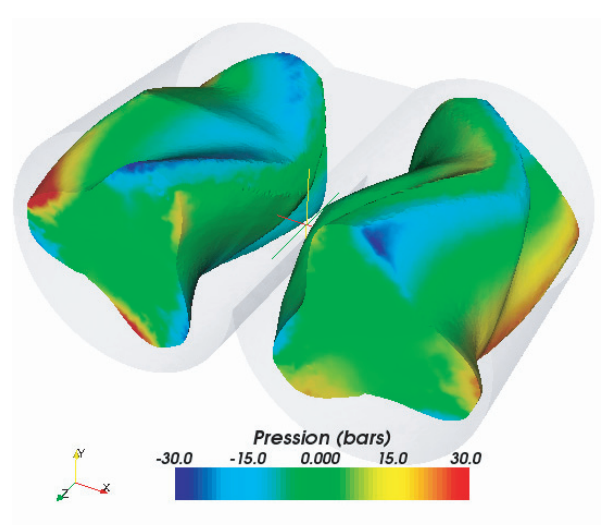

A tracer entre 0 et +60

Fig. 4. Carte du champ de pression calculé à la surface des rotors du mélangeur interne.

\section{Applications}

Trois exemples d'application aux procédés de mélange des méthodes développées sont présentés dans la suite. Il s'agit tout d'abord du calcul des champs de vitessepression dans un mélangeur interne, puis de l'étude microscopique de la dispersion d'un agglomérat de particules sous l'effet d'un cisaillement de sa matrice fluide et enfin d'une approche couplée dans laquelle une loi cinétique de dispersion est introduite dans le calcul macroscopique.

Dans chaque cas, on présente les particularités de chaque approche relativement à la méthode générale décrite aux paragraphes précédents.

\subsection{Champs de vitesse-pression dans un mélangeur interne}

La figure 1 montre l'isosurface à zéro de la fonction distance des rotors d'un mélangeur interne (définis par maillages surfaciques STL d'environ 6000 nœuds) immergés dans le maillage de calcul qui est composé d'environ 180000 nœuds et 1 million d'éléments.

Dans ce cas, les conditions de mouvement de rotation sur les objets solides sont imposées aux limites du domaine mais aussi dans l'ensemble des nouds du maillage de calcul appartenant aux éléments intérieurs aux rotors, dans le but de libérer le calcul d'un grand nombre de degrés de libertés. La distance entre les deux axes de rotation des rotors vaut $43 \mathrm{~mm}$, leur vitesse de rotation vaut respectivement 1,2 tr.s $\mathrm{s}^{-1}$ et $1,8 \mathrm{tr} . \mathrm{s}^{-1}$ (les deux rotors tournent en sens opposé) et la viscosité du fluide vaut 100 Pa.s.

La simulation, lancée sur 16 nœuds d'un cluster de machines SUN X4100, Opteron 280 à 2,4 GHz, a nécessité environ $1 \mathrm{~min}$ de calcul total pour une position donnée des rotors.

Les figures 4 et 5 montrent respectivement le champ de pression obtenu sur les surfaces des rotors et une carte

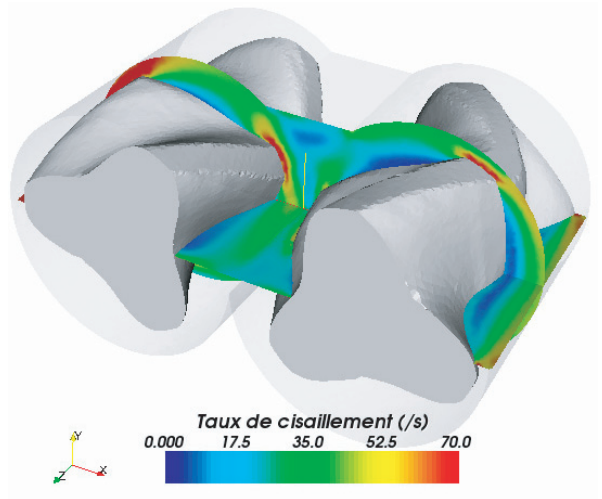

Fig. 5. Cartes du champ des taux de cisaillement calculé, sur différents plans de coupe du domaine fluide.

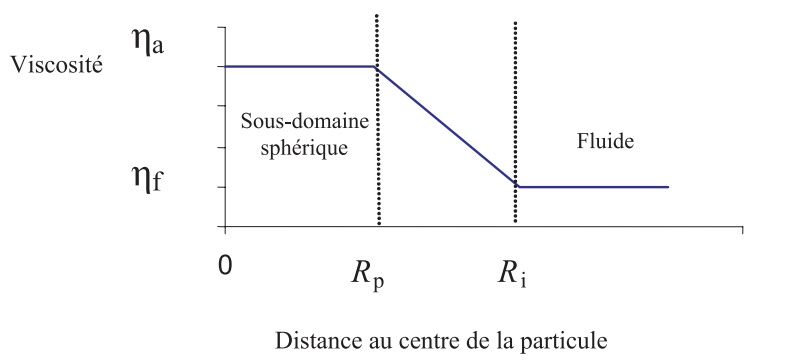

Fig. 6. Représentation de la viscosité dans le cas d'un sousdomaine sphérique immergé dans un fluide $\left(R_{\mathrm{p}}\right.$ est le rayon de la particule, $R_{\mathrm{i}}$ le rayon de la zone d'interaction).

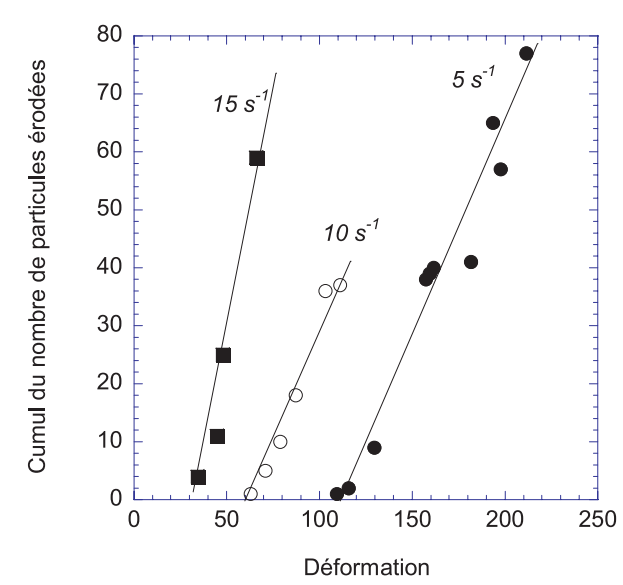

Fig. 7. Cumul du nombre de particules érodées pour un agglomérat de rayon $150 \mu \mathrm{m}$ soumis à un écoulement de cisaillement simple dans un entrefer de $0,5 \mathrm{~mm}$. Simulation réalisée pour trois taux de cisaillement différents 5,10 et $15 \mathrm{~s}^{-1}$.

des taux de cisaillement obtenus dans deux plans de coupe perpendiculaires du domaine fluide. On constate sur la figure 4 que la pression est maximale en amont de la pale et minimale après son passage. Elle est plus forte lorsque la pale est proche de la paroi et est également plus importante sur le rotor de gauche, qui a la vitesse la plus élevée. Ceci est confirmé sur la figure 5, qui montre que les taux 


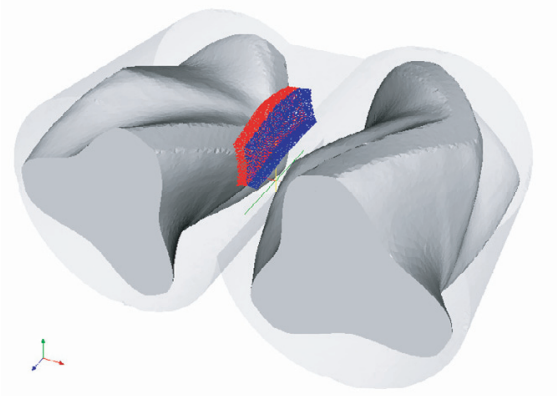

a

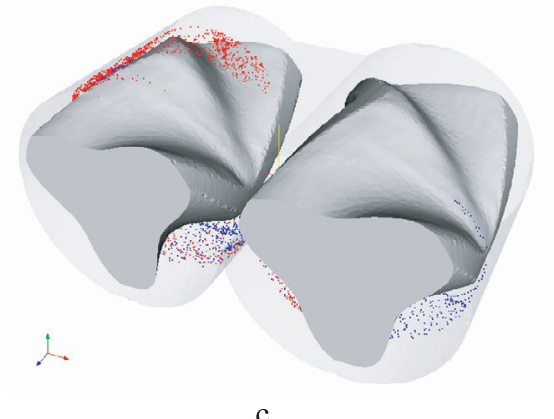

c

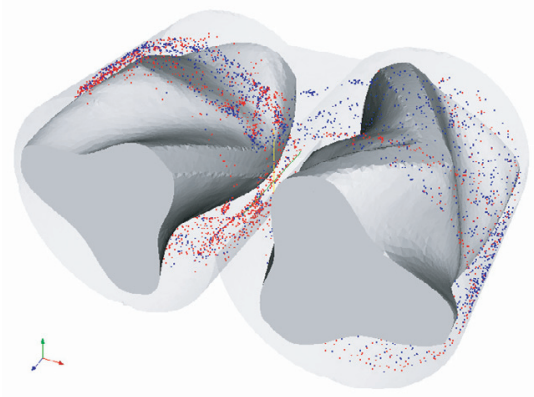

e

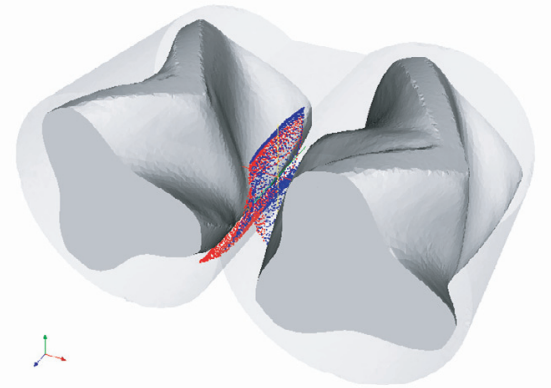

b

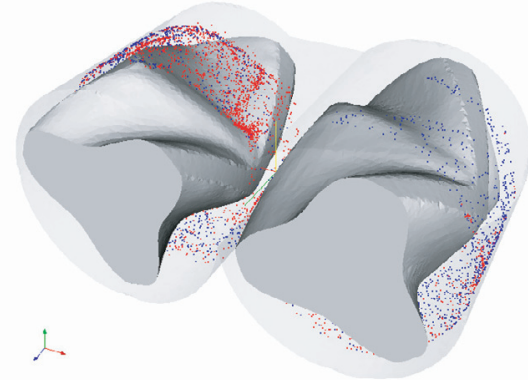

d

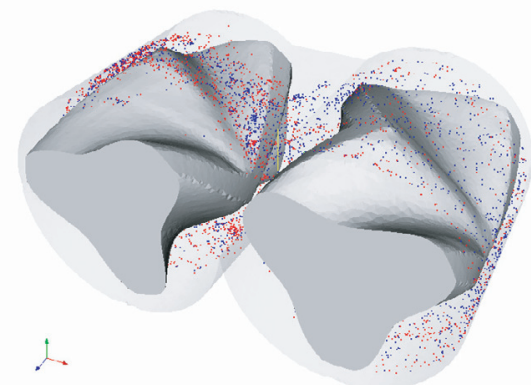

$\mathrm{f}$

Fig. 8. Calcul du lâcher d'un ensemble de 2000 particules dans un mélangeur interne. État de distribution après 0 s (a), $0,1 \mathrm{~s}$ (b), $1 \mathrm{~s}(\mathrm{c}), 2 \mathrm{~s}(\mathrm{~d}), 3 \mathrm{~s}$ (e) et $4 \mathrm{~s}(\mathrm{f})$.

de cisaillement sont maxima dans les entrefers au-dessus des pales, et plus forts à gauche qu'à droite. La zone centrale, où la distance entre les deux rotors est importante, est le siège de taux de cisaillements plutôt faibles.

\subsection{Dispersion d'agglomérats sous cisaillement}

Nous présentons ici une modélisation directe 2D de la dispersion de charges sphériques dans une matrice polymère soumise à un cisaillement. Le détail des calculs ainsi qu'une application à un écoulement 3D sont donnés dans [10]. L'agglomérat est considéré comme un assemblage de sphères rigides, liées entre elles par une énergie de cohésion. Nous prenons donc en compte dans ce calcul un ensemble de domaines rigides tel que défini par l'équation (1). La cohésion est assurée par une viscosité artificielle de cohésion $\eta_{l}$ qui évolue linéairement sur une bande de recouvrement autour des sphères, entre le rayon de la sphère $R_{\mathrm{p}}$ et un rayon d'interaction $R_{\mathrm{i}}$, d'une valeur de viscosité $\eta_{\mathrm{a}}$ très élevée à la valeur de la viscosité du fluide (Fig. 6).

La figure 2 montre l'évolution, à différents temps, d'un agglomérat de rayon initial $150 \mu \mathrm{m}$ formé de 178 particules, soumis à un taux de cisaillement de $5 \mathrm{~s}^{-1}$ dans un entrefer de $5 \mathrm{~mm}$. Un phénomène d'érosion est mis en évidence par cette simulation : lorsque les particules en périphérie de l'agglomérat ne sont plus sous l'influence de leurs voisines, elles se détachent de l'agglomérat et sont entraînées par l'écoulement. Le nombre de particules détachées croît linéairement avec le temps pour un taux de cisaillement donné, c'est-à-dire en fait avec la déformation (Fig. 7). On montre aussi, sur cette figure, que la cinétique d'érosion dépend du taux de cisaillement. Ces résultats sont en accord qualitatif avec la loi simple déduite des expériences de [11] et [12], qui peut se traduire par l'expression :

$$
\frac{\mathrm{d} r}{\mathrm{~d} t}=-\frac{K \dot{\gamma}}{r^{2}}
$$

où $r$ est le rayon de l'agglomérat, $\dot{\gamma}$ le taux de cisaillement et $K$ une constante dépendant du matériau. 

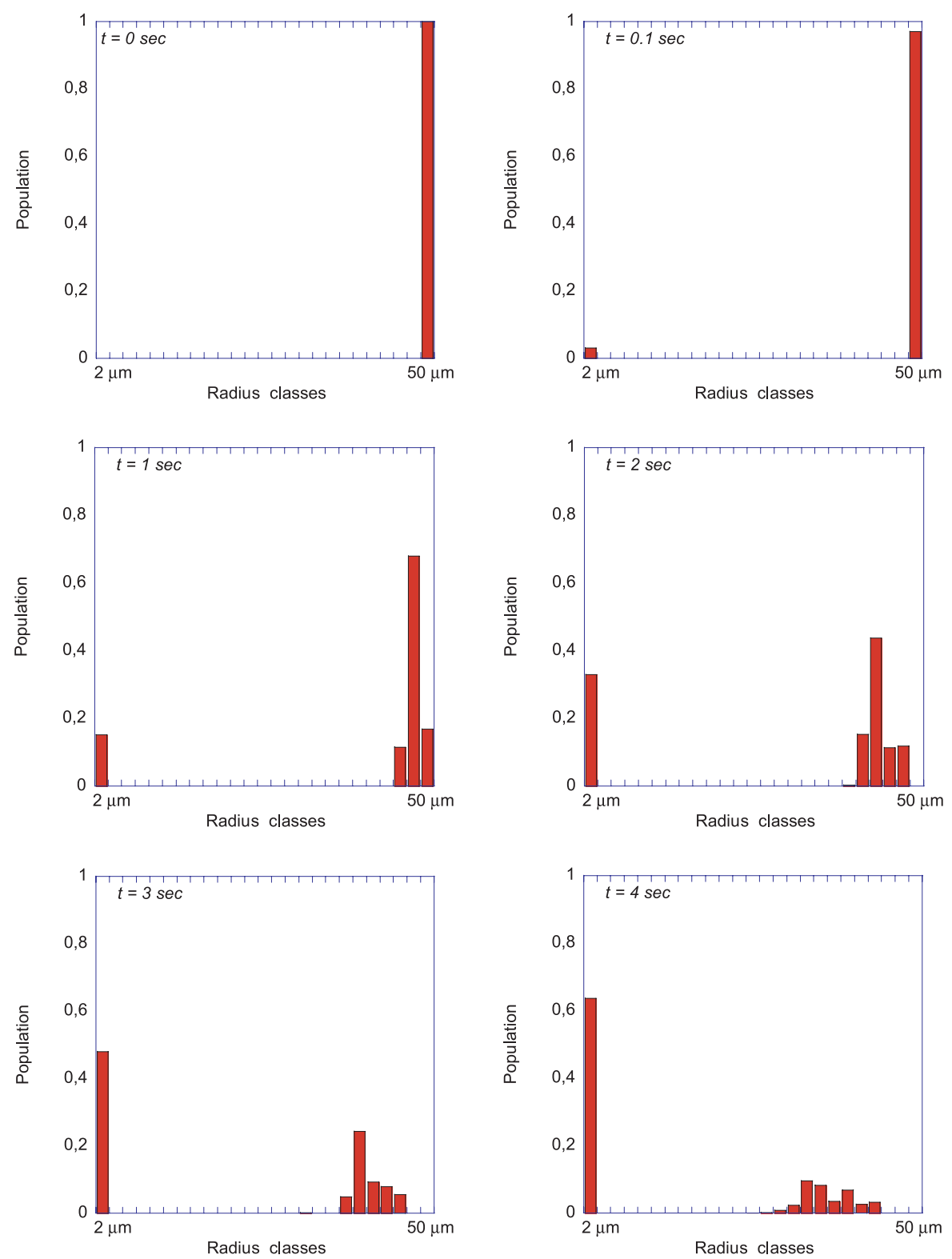

Fig. 9. Histogrammes de densité massique de distribution de rayons de particules pour les classes comprises entre $2 \mu \mathrm{m}$ et $50 \mu \mathrm{m}$ à différents instants : $t=0 \mathrm{~s}, 0,1 \mathrm{~s}, 1 \mathrm{~s}, 2 \mathrm{~s}, 3 \mathrm{~s}$ et $4 \mathrm{~s}$.

Ce type de modèle peut donc permettre, après implémentation de forces interparticulaires plus réalistes, de définir numériquement, dans des écoulements plus ou moins complexes, des lois d'évolution macroscopiques du type de l'équation (3), que l'on pourra ensuite, comme c'est fait dans la section suivante, coupler avec des modèles d'écoulement macroscopiques.

\subsection{Modélisation multi-échelle dans un mélangeur interne}

Dans cette partie, on applique directement le modèle cinétique donné par l'équation (3) dans l'écoulement présenté à la section 6.1 . On utilise une technique de lâcher de 2000 particules dans l'écoulement macroscopique. Ces 2000 particules sont supposées représenter un échantillon significatif des agglomérats initialement présents dans le mélangeur, que l'on localise dans la région de faible cisaillement située entre les deux rotors (Fig. 8a).

À chaque pas de temps (ici de $0,01 \mathrm{~s}$ ), on calcule par déplacement lagrangien les nouvelles positions des particules, ainsi qu'une interpolation du champ de taux de cisaillement à leur position, afin de calculer l'évolution de leur rayon grâce à l'équation (3).

La figure 8 montre les positions respectives des particules introduites, pour différents temps au cours de la rotation des rotors. La moitié des particules étant initialement colorés en rouge et l'autre en vert, cette figure illustre l'efficacité du mélange distributif du procédé. Au 
départ, la majorité des particules est entraînée par chacun des rotors, puis le mélange commence à se faire à travers la zone d'interpénétration, pour arriver, après seulement $4 \mathrm{~s}$, à un certain niveau de dispersion.

On peut également caractériser au cours du temps l'évolution du mélange dispersif. Pour cela, on considère que chacun des 2000 agglomérats initiaux a un rayon de $50 \mu \mathrm{m}$ et est constitué de particules élémentaires de rayon $2 \mu \mathrm{m}$. On considère uniquement un mécanisme d'érosion, arrachant aux agglomérats des particules élémentaires, contrôlé par l'équation (3). À chaque pas de temps, chaque agglomérat voit donc son rayon évoluer, en fonction du taux de cisaillement auquel il a été soumis. Les différentes tailles d'agglomérats sont regroupées en 25 classes, allant de 2 à $50 \mu \mathrm{m}$. Au départ, tous les agglomérats sont dans la classe $50 \mu \mathrm{m}$ (Fig. 9a). Ensuite, au cours du temps, cette classe diminue, au profit de classes de tailles plus faibles (30 à $45 \mu \mathrm{m}$ ), mais surtout de la classe $2 \mu \mathrm{m}$, qui recueille toutes les particules érodées. La figure 9 montre les histogrammes de densité massique de distribution de rayons de particules à différents instants, c'est-à-dire l'efficacité du mélange dispersif. Dans ce calcul, le coefficient $K$ a été pris arbitrairement à $2 \mu \mathrm{m}^{3}$.

\section{Conclusion}

Une approche par les techniques d'interaction fluidestructure a permis de simuler, à différentes échelles, différents phénomènes mécaniques intervenant dans un mélangeur interne. Ces simulations montrent qu'il est possible d'obtenir des résultats à l'échelle microscopique, qualitativement en accord avec l'observation expérimentale. À l'échelle macroscopique du procédé, il est ainsi possible de prédire, par des techniques rapides, robustes et sans remaillage, les champs de vitesse-pression dans un mélangeur interne. On peut aussi, par des techniques de lâcher de particules couplées à un modèle cinétique, caractériser la qualité de mélanges dispersif et distributif dans une géométrie complexe.

Différentes perspectives à ces travaux sont à explorer : d'une part, l'application à la modélisation 3D de dispersion d'agglomérats dont les forces de cohésion sont dérivées d'un potentiel de type Lennard-Jones, plus réaliste dans le cas de solides. D'autre part, l'extension à l'échelle macroscopique des théories cinétiques mixtes d'érosion-rupture dans un formalisme eulérien, permettant de décrire l'état de mélange en tout point du domaine fluide.

\section{Références}

[1] R. Glowinski, T.-W. Pan, T. Helsa, D. Joseph, A distributed Lagrange multiplier/fictitious domain method for particulate flows, Int. J. Multiphase Flow 25 (1998) 755794

[2] P. Singh, D. Joseph, Sedimentation of a sphere near a vertical wall in an Oldroyd-B fluid, J. Non-Newt. Fluid Mech. 94 (2000) 179-203

[3] H. Hu, Direct simulations of flows of solid-liquid mixtures, Int. J. Multiphase Flow 22 (1996) 335

[4] R. Glowinski, T.-W. Pan, T. Helsa, D. Joseph, J. Periaux, A fictitious domain approach to the direct numerical simulation of incompressible viscous flow past moving rigid bodies: Application to particulate flow, J. Comput. Phys. 169 (2000) 363

[5] P. Schneider, D. Eberly, Geometric Tools for Computer Graphics, Morgan Kaufmann series in Computer Graphics, San Francisco, 2002

[6] N. Patankar, P. Singh, D. Joseph, R. Glowinski, T.-W. Pan, A new formulation of the distributed Lagrange multiplier/fictitious domain method for particulate flows, Int. J. Multiphase Flows 26 (2000) 1509-1524

[7] P. Laure, G. Beaume, O. Basset, L. Silva, T. Coupez, Les méthodes numériques pour les écoulements de fluides chargés, Proc. $1^{\text {er }}$ Colloque du GDR Interactions FluideStructure, 2005

[8] O. Basset, Simulation numérique d'écoulement de fluides hétérogènes sur grille de calcul. Ph.D. thesis, École Nationale Supérieure des Mines de Paris, Paris, 2006

[9] H. Digonnet, T. Coupez, Object-oriented programming for « fast-and-easy » development of parallel applications in forming processes simulation, in K.J. Bathe, (ed.), Second MIT Conference on Computational Fluid and Solid Mechanics, 2003, pp. 1922-1924

[10] M. Lebøuf, T. Coupez, N. Ghamri, B. Brulé, B. Vergnes, Simulation directe 2D et 3D de la dispersion d'agglomérats sous cisaillement dans une matrice polymère, Proc. $18^{\mathrm{e}}$ Congrès Français de Mécanique, 2007

[11] S.V. Kao, S.G. Mason, Dispersion of particles by shear, Nature 253 (1975) 619

[12] V. Collin, E. Peuvrel-Disidier, Dispersion mechanisms of carbon black in an elastomer matrix, Elastomery, 9 (2005) $9-15$ 